\title{
Fast guesses in choice reaction time'
}

ROBERT OLLMAN

UNIVERSITY OF PENNSYLVANIA

A model which describes the effect fast guesses must have on observable choice latencies and probabilities is developed, strengthened, and tested with encouraging results. With the model, it is possible to estimate "true" decision times and probabilities without requiring error-free performance in discriminative reaction time.

How do Ss exchange choice accuracy for speed? Hick (1952) proposed some choices are made by a serial dichotomization process that progresses until one alternative remains; if required to be fast, Ss might sometimes go only far enough to eliminate some alternatives and then choose randomly from the remainder. Assuming each dichotomization takes a unit time and yields an average of one bit of information, he incorrectly concluded that the relation between average response time and average information transmitted by a response must be linear.

The correct course is to postulate probability distributions over (1) the number of dichotomizations the $S$ is willing to make, (2) the outcomes of the tests. "Guessing" probabilities over the responses are also needed. If there are two choice alternatives, the complex model simplifies: the $S$ either does or does not make one test. Failure to test is equivalent to guessing.

Let $Q$ be the probability he tests; $1-Q$ is the probability he guesses instead. Given he tests, leta $a_{i j}$ be the conditional probability of making response $j$ given that stimulus $\mathrm{i}$ was presented; $\mathrm{H}_{\mathbf{i j}}$ is the corresponding mean time. If he guesses, he (pre) selects response $\mathrm{j}$ with probability $b_{j} ; K_{j}$ is its mean "trigger" time. The observable conditional response probabilities $p_{i j}$ and mean times $F_{i j}$ are related to the underlying ones by

$$
p_{i j}=Q a_{i j}+(1-Q) b_{j} \quad \quad \sum_{j=1}^{2} a_{i j}=1
$$

$$
F_{i j}=\frac{Q a_{i j} H_{i j}+(1-Q) b_{j} K_{j}}{Q a_{i j}+(1-Q) b_{j}} \quad \sum_{j=1}^{2} b_{j}=1 \quad j=1,2
$$

The overall mean response time $F=\underset{i}{\Sigma_{\pi}}\left(S_{i}\right) \sum_{j} p_{i j} F_{i j}$ is a linear function of $Q$, but $T(S, R)$, the average information transmitted per response, which involves terms of the form $p_{i j} \log _{2} p_{i j}$, is not; hence the relation between $F$ and $T(S, R)$ that is swept out as $Q$ assumes various values is nonlinear.

It is in the spirit of Hick's proposal that (1) the test is unbiased $\left(a_{11}=a_{22}=a=1-a_{12}=1-a_{21}\right),(2)$ choice time depends on neither the stimulus nor the response $\left(\mathrm{H}_{12}=\mathrm{H}_{21}=\mathrm{H}_{11}=\mathrm{H}_{22}=\mathrm{H}\right)$, (3) the guessing times $\mathrm{K}_{\mathrm{j}}$ are independent of how the guesses are distributed over the responses, (4) $K_{1}=K_{2}=K$, and (5) guesses are fast $(\mathrm{K}<\mathrm{H})$.

The decision probability a will, and the decision time $H$ may, depend on the stimulus similarity, but the guessing parameters $b_{j}$ and $K$ should not. The guessing probability $1-Q$ will depend on the speed incentives, and so may the distribution of guesses over the responses, $b_{j}$, but the decision parameters $a$ and $H$, and the guess time, $K$, should not. Note the guess time, $K$, is to be affected by neither variable.

Method

Three hired male Ss were run individually in a sound attenuating chamber. Stimulus tones of $100 \mathrm{msec}$.duration were delivered to the right pad of PDR-8 earphones. The tones were perfectly detectable, but members of the pairs used were similar enough $(900,907)$ and $(900,920)$ cps to be confusable. In each stimulus condition, a stimulus randomly selected from the relevant pair was presented every $2.5 \mathrm{sec}$. without explicit warning. A Hewlett-Packard counter-printer system recorded all events and times.

So they would not guess to be fast, Ss were not at first told that their judgments were being timed; they were paid to be accurate. Having worked under accuracy incentives in both stimulus conditions, they were told of the timer and that henceforth payment would also depend on judgmental speed: one point was to be subtracted from the symmetric payoff matrix if a judgment took more than $X$ sec. to render. Judgment deadlines of $\mathrm{X}=.3, .4$, and .5 sec. were used; a single fixed deadline was in effect during a run. Information feedback was provided.

The possible speed-incentive and stimulus-confusability combinations were conducted in a haphazard but not carefully randomized order. The first 110 trials after a condition change and the first 10 trials of a run were discarded.

\section{Results}

The model predicts two linear relationships between observables will be swept out as $Q$ assumes various values:

$$
\mathrm{n}_{11} \mathrm{~F}_{11}+\mathrm{p}_{22} \mathrm{~F}_{22}=\mathrm{m}_{1}\left(\mathrm{p}_{11}+\mathrm{p}_{22}-1\right)+\mathrm{K}
$$

$$
p_{12} F_{12}+p_{21} F_{21}=m_{2}\left(p_{11}+p_{22}-1\right)+K
$$

where $F_{i j}$ and $p_{i j}(i=1,2 ; j=1,2)$ are the observable mean times and response probabilities. The slope parameters $m_{1}$ and $m_{2}$ depend only on $a, H$, and $K$; the intercept parameter $\mathrm{K}$ is the mean guess time. For- 
tunately, this prediction does not require that $b_{j}$ remain constant as speed incentives vary; it appears that $b_{j}$ did in fact fluctuate.

The model gives rise to two simultaneous equations, quadratic respectively in a and $\mathrm{H}$, with coefficients that are functions of $\mathrm{m}_{1}, \mathrm{~m}_{2}$, and $\mathrm{K}$. Solving them yields:
(3) $a=\frac{\left(m_{1}-K\right)}{\left(m_{2}+m_{1}\right)}$ or $1 / 2$
$\mathrm{H}=\frac{\mathrm{m}_{1}-\mathrm{m}_{2}}{2}$ or $\mathrm{K}$.

Figure 1 plots the data in accord with equation 2, for Ss 1 and 3. S 2 was insufficiently responsive to speed incentives to support estimation; his data are omitted.

The data points obtained without explicit speed incentives were first removed from the data, then straight lines were fit by minimizing the average square error with respect to $m_{1}, m_{2}$, and $K$. The omitted points, the rightmost on the graphs, tend to lie above the lines, which is reasonable. With $m_{1}, m_{2}$, and $K$ known, the parameters a and $H$ were calculated. Their values appear on Fig. 1.

In three of four cases, $a$ is within $5 \%$ of the probability of a correct response observed in the absence of speed incentives. For S 1, the more difficult discrimination apparently took more time to make; for $\mathrm{S}$ 3 , the times are equal. For $S 1, K$ is essentially invariant across stimulus conditions; it is not for $S 3$. Overall, the results are sensible, but better for $S 1$ than for $\mathrm{S} 3$.

\section{0 iscussion}

If this model is correct, it is possible to estimate the "true" decision probabilities and times without requiring either error-free or "pure" data. The outcome of the test is encouraging, but not definitive: that no premature responses were observed does not preclude the possibility that instead of making "'stimulus trig-

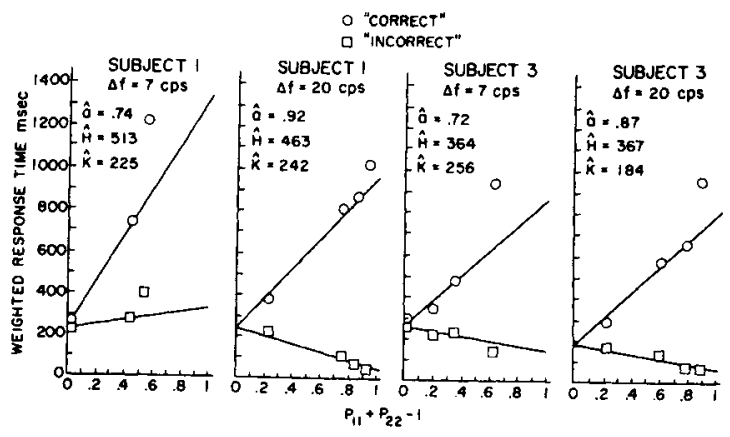

Fig. 1. Constant stimulus condition speed-accuracy exchange relations for Ss 1 and 3 with two levels of stimulus confusability. Theoretical lines, described by Equation 1, were fit by least squares.

gered" fast guesses, Ss made "prescheduled" preselected responses in order to be fast. Had they done so, the outcome of the experiment might not have been much different. The introduction of catch trials to the choice reaction time paradigm would be telling on this point.

Probably, Ss can both anticipate and guess. Introduction of catch trials will make anticipating riskythus leaving guessing as the sole "safe" way to be fast. Introduction of catch trials might thereby make this model look better than it does now, and should definitely be used in future experiments of this kind.

\section{Reference}

Hick, W. E. On the rate of gain of information. Quart. J. exp. Psychol., 4, 1952, 11-26.

\section{Note}

1. This research was supported in part by National Science Foundation grant NSF GB-1462. I wish to thank R. Duncan Luce for several helpful comments and suggestions. 